\title{
Una Experiencia de Lectura y Construcción de Conocimientos Culturales Utilizando el Sistema de Autoría "Elo"
}

\section{Reading and Building Gultural Knowledge Experience Using the Elo Authoring Software}




\title{
RESUMEN
}

El objetivo de este trabajo es mostrar algunos resultados obtenidos en una experiencia de lectura mediada por computadoras realizada por alumnos-profesores en la etapa inicial de sus prácticas de enseñanza en lengua española, donde se utiliza el sistema de autoría ELO. La lectura, que trataba sobre la importancia de aprender la lengua española, motivó también que fueran revisados algunos conocimientos sobre el mundo hispánico en general. La aplicación a posteriori de un cuestionario con preguntas de selección múltiple, mostró una elevada recuperación de la información trabajada. Se evidenció una elevada atención y satisfacción con la realización de la tarea, así como una participación activa ante la retroalimentación proporcionada. A pesar del fuerte efecto Hawthorne observado en la situación analizada, podemos inferir que actividades de ese tipo con el programa "ELO" son positivas y de buen provecho en la adquisición de conocimientos diversos y de estrategias más profundas de lectura.

Palabras clave: Lectura mediada por computadoras, Cultura hispánica, formación de profesores.

\begin{abstract}
The aim of this paper is to show some results obtained in an experience of computer-mediated reading instruction using the ELO authoring software, carried out with preservice teachers at the initial stage of Spanish Language Teaching Practices. The reading that was about the importance of learning the Spanish language, motivated that some general knowledge on the Hispanic world were also reviewed. A posttest questionnaire with questions of multiple choice, showed a high recovery of the worked information. It was evident a high attention and satisfaction with the accomplishment of the task, as well as an active participation with the feedback provided. In spite of the strong Hawthorne effect observed in the analyzed situation, we can infer that reading activities with ELO authoring software may be considered positive and may promote the acquisition of various knowledge and deeper reading strategies.
\end{abstract}

Keywords: Computer-Assisted Reading Instruction, Teaching Hispanic Culture, Language teacher education.

VÍRSIDA, Gonzalo Enrique Abio . Una Experiencia de Lectura y Construcción de Conocimientos Culturales Utilizando el Sistema de Autoría "Elo". Informática na Educação: teoria \& prática, Porto Alegre, v.8, n.2, p.65-81, jan.jun. 2006. 


\section{Introducción}

“¡Bien! ¡Muy bien!” Esa exclamación es dicha con entusiasmo por "Pipo", un personaje de CD-Roms educativos, cuando las tareas propuestas son bien hechas. Con él, mis hijos muchas veces practicaron diversas habilidades en la computadora, además de tomar contacto jugando con la lengua española. Tal es así, que en mi familia, muchas veces cuando hacemos algo bien, todavía nos recordamos e imitamos a Pipo exclamando esas frases de aprobación en español, a pesar de que nuestra comunicación normalmente transcurre en portugués.

No es de Pipo que quiero tratar en este trabajo, pero él nos indica la importancia de la presencia de otra persona - o personaje -, que nos ayude, estimule y oriente en el dominio de nuevos conocimientos y habilidades. La indicación del personaje de que la tarea pretendida era realizada con éxito, cosa que ocurría a través de la retroalimentación o feedbackque él proporcionaba, es a nuestro juicio, un detalle esencial en ese proceso.

En los tiempos actuales, donde la comunicación es prácticamente instantánea, la inmediatez de la vida cotidiana se hace cada vez más ostensible. A modo de ejemplo: una respuesta a un correo electrónico ya no es bien vista si demora más de veinticuatro horas. Por eso, los sistemas automáticos de soporte al aprendizaje son y están cada vez más sintonizados con las nuevas realidades que nos circundan.

Una interacción hombre-máquina-como en el caso indicado con el personaje Pipo-, es un ejemplo de como los letramientos ${ }^{1}$ pueden cambiar y adquirir nuevas formas. Esas formas deben ser estudiadas con urgencia, pues estamos ante fenómenos que no pasan de las dos décadas de existencia y con aun menos tiempo de su masificación. Por otro lado, eso también nos muestra como la mediación de las computadoras y los programas que las dirigen, puede ayudar en la adquisición de esos nuevos letramientos y de las habilidades de lectura necesarias.

Hay diversos estudios que describen deficiencias en las habilidades de lectura crítica y profunda de los textos, por parte de los alumnos que llegan a la universidad (DI STEFANO, 2000; SAMPAIO y SANTOS, 2002 entre otros), habilidades ésas, que son imprescindibles para un adecuado aprovechamiento de los estudios en la educación superior. Como fue mostrado por Carlino (2003a y 2003b), esas deficiencias no deben ser atribuídas sólo a una ineficaz o insuficiente formación escolar previa y sí a las necesidades provocadas por las prácticas de lectura actuales y los requerimientos de lectura en ese nivel educativo. Por eso, las acciones que sean tomadas, no deben ser sólo como algo remedial o paliativo de una deficiencia anterior y sí como algo propio que debe ser realizado en ese nivel.

Junto con los profesores, los programas informáticos específicos pueden jugar un importante papel en ese sentido. Según Garrett (1991), "la comprensión de lectura es la habilidad para la cual las computadoras están mejor preparadas", "los programas pueden ser diseñados para promover el uso de una gran variedad de estrategias, como son el skimming, el scanning, las inferencias, predicciones y otros, que refuerzan las estrategias de lectura ascendentes y descendentes (apud Salaberry, 2001, p. 51). Aunque el propio Salaberry comenta que esa cita pudiera ya ser considerada anticuada debido al rápido desarrollo tecnológico, los argumentos son correctos y continúan siendo válidos.

En este trabajo, mostraremos algunos resultados preliminares obtenidos en una experiencia de lectura mediada por computadoras, donde fue utilizado el programa "ELO" 
(Enseñanza de Lenguas On-line), - un sistema de autoría desarrollado en la Universidade Católica de Pelotas (UCPel) por Vilson Leffa y su equipo (ver LEFFA, 2003a y 2005). Los resultados de este estudio descriptivo-exploratorio, podrán ser utilizados en cursos de formación de profesores tanto presenciales como virtuales y en el diseño de otras investigaciones sobre el tema.

Antes de pasar a la explicación de la actividad realizada y los resultados observados, queremos comentar brevemente sobre dos asuntos que consideramos convenientes: el letramiento electrónico, así como otras experiencias con el uso de diversos sistemas informáticos de ayuda a la lectura.

\subsection{Nuevos letramientos y letramiento digital}

Las nuevas formas de letramiento o "new literacies" exigen habilidades para leer y escribir tanto en el formato papel como en el digital, y la definición de letramiento se está ampliando para incluir "las aptitudes, estrategias y habilidades necesarias para poder usar con éxito y adaptarse a los rápidos cambios en los contextos y las tecnologías de la información y de la comunicación, que continuamente emergen e influyen sobre todas las áreas de nuestras vidas personales y profesionales. Esos nuevos letramientos nos permiten usar la Internet y las otras TICs para identificar preguntas importantes, encontrar informaciones, evaluar críticamente la utilidad de esas informaciones y poder resumirlas, para responder a esas preguntas y después, comunicar las respuestas a los demás" (LEU, 2000, p. 476; LEU et al., 2004, p. 1572). ${ }^{2}$

El fenómeno de la hipertextualidad, aunque no es exclusivo del letramiento digital, está muy relacionado con las nuevas tecnologías de la información y la comunicación (TICs) basadas en la Internet. ${ }^{3}$ En esa unión en- tre lectura y tecnología en la Internet, - de forma contraria a lo que muchos puedan pensar-, la importancia de los profesores aumenta, pues a ellos les toca organizar experiencias de aprendizaje dentro de ambientes de información que son más ricos y complejos (LEU etal. p. 1589).

Selfe (1989), propone una analogía entre la adquisición del letramiento electrónico y la adquisición de una segunda lengua, por lo que también, existirán problemas de interferencia. De hecho, apoyado en el conocimiento previo es posible formular hipótesis sobre las maneras de interaccionar con la máquina y con el texto electrónico, aunque ese proceso sea parcial y no totalmente transferible. La autora propone un modelo de letramiento en capas, donde existen capas gramaticales de dos tipos diferentes. Una, es aquella que abarca las nuevas convenciones asociadas a los antiguos elementos de la escritura alfabética transpuestos para el contexto electrónico, mientras que la otra, es la que se refiere a los diferentes conjuntos de normas asociados a las etapas superiores del letramiento electrónico.

Buzato (2001), al estudiar, de forma colaborativa a "Teresa" (una profesora de lengua extranjera caracterizada como semi-letrada electrónica), evidenció tres clases de problemas relacionados con las capas gramaticales que se sobreponen al letramiento alfabético en el proceso de adquisición del letramiento electrónico, y que pueden funcionar como "gatillos" que favorecen el surgimiento de la sensación de impotencia del usuario frente a las demandas de lectura o escritura en el ambiente cibernético.

A continuación, una de las opiniones representativa de los problemas por los cuales pasó "Teresa":

Miré y no sabía qué tenía que hacer. ¿Sabe? era como cuando uno tiene una barrera 
en su frente. ¡Son tantas opciones! y es estraño que de repente uno no consigue leer. Es como sentirse un analfabeto de verdad. ¿Sabe que yo no conseguía leer? Tal es así, que yo tenía el texto aquí (indica a la pantalla) y leía y no entendía (traducción mía).

Como concluye Buzato (op. cit.) en su trabajo sobre el caso "Teresa", esa caracterización de los problemas generadores de ansiedad en el proceso de familiarización del profesor con la lectura o escritura electrónica, abre el espacio para una discusión más amplia sobre las necesidades que deben ser contempladas en los programas de formación de profesores, en lo que respecta a la inserción de las computadoras en las prácticas de enseñanza de lengua extranjera.

\subsection{Programas de aprendizaje y de apoyo a la lectura mediados por computadoras}

Veamos, inicialmente, algunos de los numerosos programas de apoyo a la lectura, que trabajan con glosarios acoplados a textos, con la idea de ayudar en la comprensión y adquisición de vocabulario.

Salaberry (2001, p. 46-47), relata las experiencias de Grace (1998) con estudiantes de francés, y el efecto benéfico en la retención de vocabulario de las anotaciones con multimedios presentes en los textos, debido, probablemente, al análisis y procesamiento más elaborado del estímulo, o sea, por el acceso a un mayor número de asociaciones. De forma similar, en el estudio de Hegelheimer (1998), los alumnos que dispusieron de más consultas a las anotaciones, son los que mostraron una mayor retención de vocabulario en las pruebas realizadas a posteriori (HEGELHEIMER y CHAPELLE, 2000, p. 47).

También, con el propósito de ayudar en la lectura independiente, en este caso con tex- tos presentes en la Internet, LeLoup y Ponterio (2005) describen dos programas que pueden auxiliar en dicha tarea, - el "Ultralingua.net" y el "WordChamp" -, los cuales introducen linksque muestran el significado de las palabras que aparecen en los textos que están siendo leídos y que pueden ser activados por el lector cuando lo necesita.

El propio proyecto "ELO", permite la utilización de uno de los tres diccionarios disponibles: portugués/portugués; español/ portugués o inglés/portugués, de los cuales el último es el más completo de los tres, con cerca de 27.000 entradas. Como es explicado por el autor, el diccionario fue introducido en el proyecto para la lectura de un texto en la pantalla de la computadora con objetivos bastante específicos. No se trata de una lectura hecha por placer, o una lectura con fines académicos. Se trata, específicamente, de la enseñanza de la lengua extranjera a través de la lectura y para la lectura. La idea es la de que el alumno pueda aprender a leer leyendo " básicamente un texto que está por arriba de su nivel de competencia lingüística y, por lo tanto, incomprensible", pero haciéndolo comprensible con la ayuda del diccionario. La atención del alumno va a estar en el texto, que es de donde él construirá el significado, y no en el diccionario (LEFFA, 2001).

Otro tipo de posibilidad, son los programas 0 actividades que presentan preguntas para verificar la comprensión lectora. Entre ellos, se puede mencionar el "Accelerated Reader" (AR), que es un "sistema de información de aprendizaje" basado en preguntas de selección múltiple. Según los autores del artículo, Topping y Fisher (2003), ese programa es muy popular en los Estados Unidos, donde se encuentra en aproximadamente la mitad de las escuelas, siendo las experiencias relatadas en el artículo, las primeras realizadas en gran escala en el Rei- 
no Unido. Esa investigación mostró que el programa tiene potencial, pero sólo si es implementado correctamente, pues se vio que algunos profesores no supieron tomar las acciones adecuadas, aunque los datos proporcionados por los informes generados por el programa indicaban que existían problemas específicos en las lecturas.

\section{El programa "RITA" ("Reader's} Interactive Teaching Asistant') es una derivación de los "Asistentes de Enseñanza Inteligentes" (ITA en inglés), específicos para auxiliar en el proceso de lectura y concebidos como una ayuda para los profesores. El programa "RITA" fue utilizado con niños que no habían respondido bien a los métodos tradicionales de intervención en la lectura. No hubo grandes diferencias como un todo cuando se comparó con los métodos tradicionales de lectura, pero sí se mostró efectivo en la parte educativa y de costos. También, fueron observados grandes avances en el interés y entusiasmo de los alumnos cuando trabajaban con las computadoras, por lo que él fue recomendado como una ayuda, sobre todo para cuando son detectados problemas de deficiencias de lectura a temprana edad (NICOLSON, FAWCETT y NICOLSON, 2000).

Los sistemas inteligentes de aprendizaje de lenguas mediados por computadoras (ICALL), a diferencia de los CALL , incorporan un procesador de lenguaje natural que es capaz de analizar las respuestas de los alumnos, comparándolas con las reglas gramaticales con el objetivo de identificar posibles problemas en las respuestas.

Un ejemplo de un sistema moderno de tutoría inteligente es el "AutoTutor", diseñado por un equipo interdisciplinario que conforma el "Tutoring Research Group"4. Ese sistema auxilia en el letramiento informático de los estudiantes, por medio de conversaciones multiturnos, donde se emplean las tácticas utilizadas por los tutores humanos. El "AutoTutor" fue probado con doscientos estudiantes en un curso de letramiento informático donde hubo avances, cuando fueron comparados con la condición control referente a los alumnos que sólo repetían la lectura del texto.

En otros estudios se ha mostrado que la eficacia del "AutoTutor" no se debe al medio de presentación utilizado (agentes animados con habla impresa y auditiva o sus alternativas), sino, principalmente, al contenido del diálogo que se va presentando (TANNER y GRAESER, 2006). Hay un intercambio de turnos de forma colaborativa que, normalmente, no es posible de implementarse en sala de aula. Además, los tutores por lo general, hacen más preguntas que estimulan el desarrollo del pensamiento profundo, introducen más problemas para resolver, y trabajan sobre más casos específicos, que los profesores que dan clases normalmente (GRAESER, PERSON y HU, 2002).

La serie de interacciones que ocurre en las sesiones de tutoración tienen normalmente dos etapas más que el clásico patrón de interacción I-R-E (Iniciación-Respuesta-Evaluación) entre profesores y alumnos, pues después de esos tres primeros pasos, el tutor y el alumno entran en una etapa de aumento de la calidad de la respuesta inicial por medio de una secuencia de turnos de habla que, por lo general, culmina con una pregunta de medición de la comprensión como, por ejemplo: “¿entendiste?” Por otro lado, los turnos de los tutores son mucho más cortos que los casi siempre largos monólogos de los profesores. También los tutores normalmente dan más recomendaciones o pistas con una especificidad progresiva y de una forma mucho más elaborada que lo que ocurre en las salas de aula. 
Aquí es de gran importancia la existencia de una retroalimentación pedagógica de calidad, pues los alumnos pueden aprender mejor cuando después de cometer un error, lo reconocen enseguida. Existe una fuerte tendencia a una "ilusión de comprensión de los textos", donde normalmente los alumnos no perciben sus errores hasta que alguien llama su atención al respecto. Es por eso que el tutor puede utilizar la retroalimentación para indicar el error y ayudar al alumno en la profundización y comprensión de los conceptos subyacentes (CHI et al., 2001, TANNER JACKSON y GRAESER, 2006).

En Zarzosa Escobedo (2004) encontramos otro ejemplo de avances obtenidos con un programa de computadora llamado "Cuestionando al autor" ("QtA"), utilizado para el desarrollo de la lectura estratégica y crítica de textos expositivos y argumentativos en el nivel universitario.

Con ese programa, que se apoya en las ideas del protocolo homónimo de Beck et al. (1997), mediante diversos tipos de preguntas se pretende que el lector alcance cuatro objetivos: (1) reconozca expresiones que sinteticen las afirmaciones del autor sin deformaciones; (2) identifique las proposiciones principales de un escrito; (3) articule y jerarquice los diferentes conceptos clave y; (4) juzgue la suficiencia y claridad de la información del texto ${ }^{5}$.

El autor explica la efectividad mostrada en las actividades evaluadas, citando una serie de elementos que fueron identificados por Swanson y Deshler (2003) como relevantes para el aprendizaje. Ellos son: La práctica explícita de lo que se quiere enseñar; instigar, por medio de una serie de claves y señales, los procesos psicológicos que correspondan con la tarea - en este caso, por medio de las preguntas proporci- onadas por el programa -; el uso de un recurso moderno de interacción como es la computadora; los ejercicios adaptados al propio ritmo del educando; la división de la tarea en pequeñas unidades accesibles y secuenciadas $\mathrm{y}$, por último, la existencia de un sistema de retroalimentación frecuente y significativo (apud Zarzosa Escobedo, 2004) ${ }^{6}$.

El programa "QtA" arriba descrito, posee más o menos la misma estructura del programa "ELO" utilizado por nosotros. En la pantalla aparece una sección con el texto que es analizado, otra con la pregunta realizada, mientras que una tercera pantalla muestra las alternativas para cada pregunta. Un cuarto espacio - a diferencia del "ELO" -, muestra las secciones en que fue dividido el texto analizado. La retroalimentación o feedback, aparece en una ventana flotante en el centro de la pantalla. Además, existen diversos botones: de selección de las preguntas, de la alternativa correcta, de aceptación de la alternativa, etc., y otro para mostrar el gráfico con el desempeño alcanzado.

Una de las ventajas del programa "ELO", es que él posee un sistema más avanzado de retroalimentación diversificada, que puede mejorar el diálogo y la interacción. Existe la posibilidad de generar tres tipos de retroalimentación. La "genérica", que es la más simple, sólo evalúa la respuesta del alumno y dice si está correcta o incorrecta. En cambio, la retroalimentación "situada" consiste en un comentario específico hecho sobre un segmento que aparece en la respuesta del alumno, simulando con más riqueza lo que puede ocurrir en una interacción frente a frente. Puede ser "correctiva", cuando la respuesta está incorrecta, o "repetitiva" cuando la respuesta está correcta y, en ese caso, la retroalimentación retoma alguna cosa de lo que el alumno escribió. Uno de los objetivos de la retroalimentación "situada", principalmente 
cuando es correctiva, es alertar al alumno hacia algunos aspectos que pudieron pasar desapercibidos ${ }^{7}$. El tercer tipo de retroalimentación pedagógica llamada de "estratégica", puede ser proporcionada al alumno no sólo en el exacto momento en que él muestra necesidad de aplicar la estrategia, como también en la cantidad exacta, pues el sistema de autoría "ELO" puede ofrecer una sucesión de pistas hasta que el alumno llegue a la respuesta deseada.

El sistema "ELO" permite también un análisis sofisticado de las respuestas proporcionadas por los alumnos, muy superior a los que podemos encontrar en otros programas de autor populares como el "Hot Potatoes", pues puede trabajar con segmentos de esas respuestas y con grados de tolerancia variable, con el fin de simular un diálogo verosímil y pedagógicamente relevante ${ }^{8}$.

En la experiencia que aquí relataremos, realizada con futuros profesores de español, optamos por la posibilidad de una actividad de lectura que posee una interferencia media por parte del profesor (LEFFA, 2003a), o sea, una lectura con preguntas de selección múltiple que permite alguna interacción debido a la presencia de un cierto grado de retroalimentación individualizada.

Para ejemplificar esto, mostramos la primera de las preguntas realizadas en español por el sistema, en el texto aqui analizado: "Basado en sus conocimientos generales, y en lo que dice el artículo en su primera parte, observe el mapa de América del Sur y diga cuál es el número de países sudamericanos donde el español es lengua oficial." El programa ofrece para elegir cuatro posibilidades (nueve, diez, once o doce países), con sólo una alternativa correcta. Ver figura 1.

Si la alternativa elegida es la correcta,

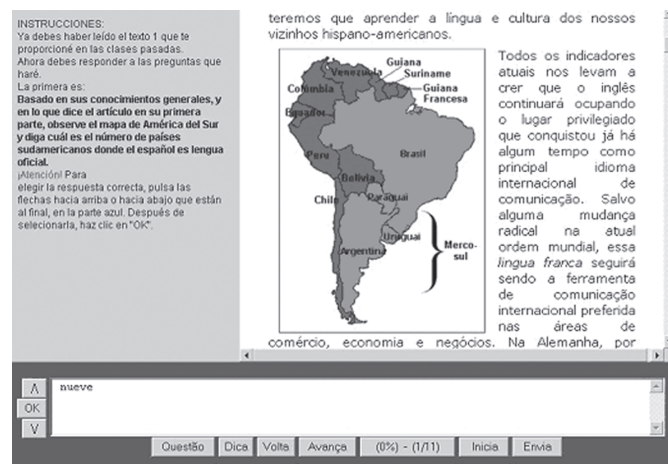

Figura 1. Pantalla del programa "ELO" mostrando una actividad de lectura con selección múltiple

aparece el siguiente mensaje con una retroalimentación positiva, donde se comenta y amplía dicha respuesta: "¡Muy bien! Son nueve: Venezuela, Colombia, Ecuador, Perú, Bolivia, Chile, Argentina, Uruguay y Paraguay. Sumados a los otros del Caribe, Centroamérica, España y uno en África (Guinea Ecuatorial), hacen un total de veintiún (21) países o repúblicas donde el español es la lengua oficial". ${ }^{9}$

Si la respuesta fuese negativa, es el siguiente mensaje el que aparece: “¿Sí? ¡Cuidado! Debes leer con más atención el primer párrafo del artículo".

Como podemos ver en el contenido de ese mensaje, su objetivo es intentar sugerir estrategias que puedan llevar al alumno a la respuesta correcta. No se da la respuesta, pero se intenta mostrar cómo llegar a ella

Si no es suficiente esa retroalimentación estratégica, probablemente el alumno accionará el botón "Dica"10, que para esta pregunta mostrará una información mayor que la proporcionada en la retroalimentación negativa previa, pero sin dar la solución textual: "Observa que en el primer párrafo son citados unos países donde NO se habla español."

Después de estas consideraciones 
generales y de introducción al funcionamiento del programa "ELO", pasaremos a describir la actividad de lectura realizada y los resultados obtenidos, que serán después comentados de forma concisa.

\section{El contexto de la actividad}

La experiencia consistió en la lectura previa del texto en portugués "Por que os brasileiros devem aprender espanhol?", de la autoría de João Sedycias (SEDYCIAS, 2005) ${ }^{11}$, a lo cual se unió un trabajo posterior en el laboratorio de informática con el programa "ELO", donde fue explorado ese texto expositivo-argumentativo ${ }^{12}$ por parte de los alumnos del cuarto año de la licenciatura en letras portugués-español de una universidad del nordeste brasileño. ${ }^{13}$

En el laboratorio, los alumnos realizaron la lectura del texto en parejas. El programa "ELO" indica el desempeño del alumno a través de varias vías: por los propios mensajes de retroalimentación de diversos tipos que fueron arriba descritos; así como por el indicador de porciento de aciertos que aparece junto con la relación entre el número de la pregunta que está siendo realizada en ese momento y el total de preguntas de la actividad (ejemplo: "100\% 1/11"), información ésta que aparece entre los botones de comando en la parte inferior de la pantalla (ver Figura 1). Además, al final de la actividad, es generado un informe detallado del desempeño, el cual puede ser impreso o enviado al profesor, vía e-mail. En los informes aparece la identificación de la persona, el tiempo general de trabajo en la actividad, así como la descripción de las elecciones realizadas para cada pregunta y también pueden ser añadidos comentarios generales con opiniones sobre la actividad.

Esos informes fueron enviados al investigador y autor de este trabajo y sirvieron como fuente de análisis del desempeño general en la actividad, así como para la identificación de las dificultades específicas. También, fueron realizadas observaciones cualitativas sobre el comportamiento general de los alumnos durante la realización de la actividad en el laboratorio. De esa forma, este estudio exploratorio se adscribe dentro de un paradigma descriptivointerpretativo que busca informaciones para entender el grado de aceptación y utilidad de las lecturas académicas en el nivel universitario con ese tipo de actividad de lectura interactiva mediada por computadoras.

El tema de la primera de las preguntas realizadas, cuyo contenido ya vimos arriba en la descripción de los tipos de retroalimentación, fue continuado, llamando también al conocimiento de mundo previo de los alumnos, esta vez por medio de una pregunta sobre los países hispanohablantes de América Central. Otra pregunta, tenía que ver con la identificación de la lengua con mayor número de hablantes y, de forma indirecta, sobre el lugar que ocupa el español por el número de hablantes en el mundo, para lo cual era necesario interpretar un gráfico de barras presente en el texto (ver Figura 2.).

Fueron realizadas otras preguntas, tratando de temas como: las premisas internas y externas para aprender español,--que conducen a una reflexión sobre la importancia de aprender esa lengua-, y también, sobre la mayor facilidad del aprendiz hablante de portugués para aprender español o vice-versa, junto con otros. Con esas preguntas se buscaba focalizar y explotar las informaciones más relevantes presentes en el texto.

Transcurridos cuarenta y dos días de esa actividad, fue realizada una prueba de comprobación, también con respuestas de selección múltiple, pero en esta ocasión, de forma individual, sobre la localización general de los países hispanohablantes y otros conocimientos trata- 


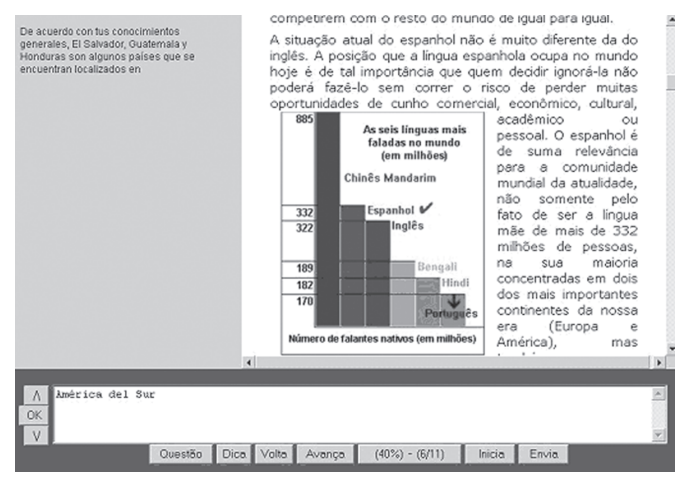

Figura 2. Fragmento de texto con gráfico de barras que debía ser interpretado para identificar la lengua con mayor número de hablantes. En la columna vertical izquierda podemos ver la sexta de las preguntas realizadas, donde se llama al conocimiento general del mundo de los alumnos para identificar los países hispanohablantes existentes en América Central. En la parte horizontal inferior aparece una de las opciones de respuestas para esa pregunta, así como los botones de funcionamiento general del programa.

dos en ese texto, cuyos resultados parciales también serán presentados aquí. ${ }^{14}$

\section{El desempeño}

\subsection{Análisis de los informes generados por el sistema "ELO".}

Hubo un total de dieciséis informes, de los cuales uno fue eliminado de los análisis debido a que era una repetición realizada por alumnos no satisfechos con el desempeño alcanzado en el primer intento. Desconsiderando el tiempo mayor de realización de la actividad (45min27seg) y el menor (6min13seg), hubo un promedio de tiempo utilizado en la actividad que fue de 14 minutos y 45 segundos.

El análisis de las respuestas correctas obtenidas en el primer intento, nos muestra donde hubo mayores dificultades. Por ejemplo: cinco de las parejas tuvieron dificultades en la quinta de las preguntas, que trataba sobre la naturaleza de los motivos para estudio del español, y donde era necesario analizar las informaciones presentes en dos largos párrafos del texto, para poder responderla. Por falta de conocimientos previos sobre la identificación de los países hispanohablantes localizados en América Central, la pregunta sexta también exhibió cinco respuestas no correctas en el primer intento.

Lo mismo ocurrió en la octava y novena de las preguntas, donde era necesario analizar varios párrafos para poder "montar" la respuesta correcta, y donde hubo cuatro faltas de aciertos en el primer intento. De cierta forma, las dos preguntas estaban relacionadas. La primera de ellas trataba sobre la aparente facilidad para entender la lengua española por parte de los hablantes de portugués, mientras que la siguiente tenía que ver con quién aprendería más fácilmente la lengua del otro, si el hablante de portugués o de español. En las dos preguntas, las respuestas probablemente se apoyaron más en las creencias previas que en las informaciones contenidas en el texto, lo cual debe haber motivado la falta de aciertos iniciales de algunas de las parejas.

Un análisis pormenorizado de las especificidades de cada pregunta y las causas posibles de los desaciertos, es algo que escapa de los objetivos descriptivos generales de este trabajo.

Como al realizar la actividad se evidenciaron dificultades con el reconocimiento y localización de algunos de los países hispanohablantes, se procedió, - una vez terminada la actividad de lectura en la computadora, a la elaboración por parte de los propios alumnos en conjunto, de una lista en español de los países hispanohablantes y su correspondiente localización geográfica general (América del Sul, América Central, Europa o África), junto con los nombres de sus capitales. Estimamos que esos conocimientos culturales son generales y 
deberían ser dominados por parte de cualquier profesional de la lengua española.

En la prueba de selección múltiple realizada a posteriori con veintinueve alumnos y de forma individual, se percibió una elevada recuperación de la información tratada en la lectura y en la actividad colaborativa que le siguió. En esa prueba, donde fue pedido que se marcase la localización general de cada país hispanohablante, sólo hubo respuestas incorrectas con Guatemala, Costa Rica y Puerto Rico (dos errores cada uno). Otro tema analizado fue el también ya mencionado, de la mayor facilidad o no, de aprender español por hablantes de portugués, lo cual fue evaluado por medio de una pregunta cerrada de "sí" o "no" y donde todos los participantes tuvieron la respuesta correcta.

\subsection{Análisis cualitativo general de la actividad}

Fue evidente que la actividad y el trabajo con las computadoras en general fue recibido de forma favorable por los alumnos. Eso fue percibido por dos vías. Una, por la observación directa de lo ocurrido en sala de aula durante la actividad, donde fueron escuchadas exclamaciones como las siguientes: "¡Menos mal! ¡Después de cuatro años estudiando aquí, por primera vez vinimos al laboratorio!", "QQué bueno esto!", “ ¡No lo creo. ¿vamos a usar las computadoras?!", etc. ${ }^{15}$

Por otro lado, fueron interesantes los comentarios emitidos de forma libre por los participantes y que fueron anexados a los informes enviados con los resultados de la actividad en el programa "ELO". Siguen algunos ejemplos, sin correcciones:

- A mí me gustó mucho esa actividad. Yo creo que es una manera diferente y agradable de fijar lo que aprendí con la lectura del texto. !Gracias profesor!
- A nosotros nos gustó mucho esta actividad. Deseamos que ese tipo de trabajo sea realizado con frecuencia. ;Hasta luego!

- Amamos la clase de hoy, pues fue muy dinámica. Esperamos tener muchas clases iguales a esta.j Gracias profesor Gonzalo!

- Fue muy bueno, porque nosotras tuvimos oportunidad de recordar la clase pasada y tener mejor conocimento geográfico.

- Esta actividad es muy legal, bien interesante y criativa. El profesor está de parabéns. Sen dúvida nosotros aprendemos mucho.

- A mi, me gustó la actividad porque és mucho mejor y proveitosa comparando-se con las otras clases.

- $A$ atividade fue mucho interesante y gostaríamos que houvese más atividades como esa. Como también nos ensinase como montar un programa como ese.

- El artículo de João Sedycias es muy relevante para nosotras estudiantes de letras porque demonstra la importancia de la aprendizaje de la lengua español para nuestro enriquecimento profesional y personal. besos.

Estos comentarios evidenciaron una aceptación y percepción de la importancia y provecho que podrían proporcionar las actividades de lectura realizadas de esa forma para el aprendizaje y formación profesional. También, constatamos el interés de algunos de los alumnos por conocer como trabajar con ese programa.

Un breve análisis lingüístico de esos comentarios muestra un detalle interesante. La palabra "actividad", en todos los casos, fue escrita corretamente en español, algo que por transferencia del portugués muy probablemente hubiera sido escrito con algún tipo de desvío. Ese hecho, estimamos que se debió, muy probablemente, a la exposición a esa palabra en español varias veces en la misma actividad de lectura.

Concatenado con lo anterior, se percibió que la actividad de lectura del texto general fue realizada con gran atención, así como la lectura 
de las preguntas, alternativas, respuestas y recomendaciones proporcionadas por el programa, y que muchos de los alumnos las estaban pronunciando y reproduciendo en voz baja. Por el aumento del input proporcionado y su captación, este hecho debe ayudar en la adquisición de la lengua española, objeto de estudio.

Los alumnos también mostraron una gran preocupación con los resultados obtenidos por ellos, aunque habíamos explicado que el objetivo de la actividad no era evaluativo. Entendemos que lo más importante era la comprensión del texto y la comprobación de las hipótesis, así como facilitar la percepción de las mayores deficiencias y de las fuentes de los errores que eran cometidos; o sea, más desde una visión de evaluación formativa y autoevaluativa. Otro de los objetivos, como ya fue dicho anteriormente, era el de proporcionar una guía para percibir los detalles más importantes de ese texto.

Por último, - - contrario a lo que pensamos que podía haber ocurrido al inicio -, no se evidenciaron grandes dudas o dificultades en la manipulación del programa y la realización de la actividad en general. Estimamos que eso ocurrió, debido a que las orientaciones que el alumno va recibiendo en la pantalla contribuyen con el entendimiento de lo que hay que hacer en cada momento.

\section{Algunos comentarios finales}

Los resultados de este trabajo no son fácilmente extrapolables y tiene algunos de los problemas de muchas de las investigaciones realizadas con enseñanza mediada por computadoras, como puede ser el pequeño tamaño de la muestra, poco tiempo para detectar efectos, etc. (SALABERRY, 1996, p. 9-10); pero estos resultados aún preliminares, por su importancia y potencial en la construcción del conocimiento general y de las competencias lectoras de los alumnos, así como por su posible contribución indirecta en la formación de los futuros profesores en la enseñanza presencial o virtual (LEFFA, 2003b), nos estimulan a continuar estudiando sobre ese tema.

De hecho, en la parte teórica y reflexiva de nuestro curso de "Prácticas de enseñanza en lengua española", ya hemos incorporado ésta y otras lecturas académicas donde se trabaja con el sistema de autoría "ELO", y también existe la intención de replicar y profundizar los estudios ya realizados ${ }^{16}$.

Hubo indicios de una lectura superficial y poco profunda, por lo menos por algunos de los alumnos. Un análisis de los tipos de preguntas y partes del texto donde eso ocurrió, así como la manera de llevar al desarrollo de operaciones y estrategias lectoras que ayuden a superar esas dificultades es algo pendiente y que merece más atención en próximos trabajos ${ }^{17}$.

Fue evidenciado un fuerte efecto Hawthorne, debido a la novedad de la exposición inicial a actividades de lectura en un laboratorio de informática, lo que para Salaberry (1996, p. 10) es otro factor que enmascara el efecto de las variables de estudio. ${ }^{18}$ De cualquier forma, la gran aceptación que tuvieron las acciones realizadas y la opinión positiva sobre la actividad de lectura en general por parte de los alumnos, es un factor importante, pues consideramos que la parte afectiva es tan importante como la cognitiva o social en el proceso educativo.

Si focalizamos el aspecto evaluativo, veremos que en la Educación a Distancia y el trabajo con computadoras en general, él es de importancia fundamental. Para GUTIERREZ y PRIETO (1994), la evaluación ideal en sistemas de enseñanza a distancia sería la autoeva- 
luación, capaz de integrar procesos y productos, de corregir y estimular el autoaprendizaje y de construir, experimentar y simular nuevos conocimientos. Creemos que un sistema de autoría como el "ELO" puede ayudar mucho en ese sentido.

\section{Referencias}

ANDERSON, J. Cognitive psychology and its implications. New York: Freeman, 1990.

BECK, Isabel L., Mckeown, Margareth G., HAMILTON, Rebecca L., KUCAN, Linda. Questioning the author: An approach for enhancing student engagement with text. Delaware: International Reading Association, 1997.

BUZATO, Marcelo. O letramento eletrônico e o uso do computador no ensino de língua estrangeira: o caso Tereza. 11ㅇ Intercâmbio de Pesquisa em Lingüística Aplicada, São Paulo, en 05/05/2001. Disponible en: <http://www.educarede.org.br/educa/internet_e_cia/ informatica.cfm?pagina=informatica_principal\&id_inf_escola=14\#>. Acceso en: 11 feb. 2006.

CÁRDENAS PÁEZ, Alfonso. Elementos para una pedagogía de la literatura. Volumen IV. Bogotá: Universidad Pedagógica Nacional, 2004.

CARLINO, Paula. Carlino, P. Alfabetización académica: Un cambio necesario, algunas alternativas posibles. Educere. Revista Venezolana de Educación, v. 6, n.20, p. 409-420, 2003a.

CARLINO, Paula. Leer textos científicos y académicos en la educación superior: obstáculos y bienvenidas a una cultura nueva. 6을 Congreso Internacional de Promoción de la Lectura y el Libro, 29 Feria del Libro, Buenos Aires, 2, 3 y 4 de mayo de 2003. XIII Jornadas Internacionales de Educación, 2003b. Disponible en: <http://www.buenosaires .gov.ar/areas/educacion/bibleduc/pdf/ paula_carlino.pdf> Acceso en: 11 feb. 2006.

CASSANY, Daniel. Tras las líneas. Sobre la lectura contemporánea. Barcelona: Editorial Anagrama, 2006.

CHI Michelene T.H. et al. Learning from human tutoring. Cognitive Science 25, p. 471-533, 2001.

DI STEFANO, Mariana. La lectura: representaciones sociales y prácticas escolares I. En portal virtual educativo del Ministerio de Educación y Cultura de la Nación, 2000. Disponible en: <http:// www.educ.ar/educar/docentes/lengua/egb2/final.jsp?url=LELI MONO 001\%2FINDEX.HTM\&nivel=3\&area=10\&tipo=165\&id=458> Acceso en: 2 feb. 2006.

ELLIS, Rod. La adquisición de segundas lenguas en un contexto de enseñanza. Análisis de las investigaciones existentes. Ministry os Education of New Zealand, 2006. Disponible en: <http:// www.sgci.mec.es/redele/Biblioteca2006/Ellis.shtml> Acceso en: 10 mar. 2006.

GASS, Susan M. Input, interaction, and the second language learner. Mahwah, $\mathrm{NJ}$ : Lawrence Erlbaum Associates, 1997.

GARCÍA PAREJO, Isabel. (Bi)alfabetismo: ¿Qué significa tener competencia lectoescritora en una segunda lengua? Glosas Didácticas, n. 15, Otoño de 2005 Disponible en: <http://www.um.es/ glosasdidacticas/GD15/gd15-05.pdf> Acceso en: 2 enero 2006

GARRETT, Nina Technology in the service of language learning: Trends and Issues, The Modern Language Journal, v. 75, n. 1, pp. 74-101, 1991.

GRACE, Caroline A. Retention of word meanings inferred from context and sentence-level translations: Implications for the design of beginning-level CALL software. The Modern Language Jornal, 82, p. 533$554,1998$. 
GRAESSER, Arthur C.; PERSON, Natalie; HU, Xiangen. Improving comprehension through discourse processes. New Directions in Teaching and Learning, 89, p. 33-44, 2002. Disponible en: <http:// internal.autotutor.org/papers/artspdfs/Halp-gp.pdf> Acceso en 9 feb. 2006.

GUTIERREZ, Francisco; PIETRO, Daniel. A Mediação Pedagógica: Educação à

Distância Alternativa. Campinas: Editora Papirus, 1994.

HEGELHEIMER, Volker. Effects of textual glosses and sentence-level audio glosses on online reading comprehension and vocabulary recall. Unpublished doctoral dissertation. Department of Educational Psychology, College of Education, University of Illinois, Urbana, IL, 1998.

HEGELHEIMER, Volker; CHAPELLE, Carol A. Methodological Issues in Research on Learner-Computer Interactions in CALL. Language Learning \& Technology, v. 4, n. 1, p. 41-59, May 2000.

LEFFA, Vilson J. Aprendizagem mediada por computador à luz da Teoria da Atividade. Calidoscópio, São Leopoldo, v. 3, n. 1, p. 21-30, 2005. Disponible en: <http://www.leffa.pro.br/trabalhos/ta.htm> Acceso en: 2 feb. 2006.

Interação simulada: um estudo da transposição da sala de aula para o ambiente virtual. In: (Org.). A interação na aprendizagem das línguas. Pelotas: EDUCAT, 2003a, p. 175-218.

. Análise Automática da resposta do aluno em ambiente virtual. Revista Brasileira de Lingüística Aplicada. Belo Horizonte: v.3, n.2, p.25 - 40, 2003b. Disponible en: <http://www.leffa.pro.br/ analise_automatica.htm> Acceso en: 12 dic. 2005.

. O uso de dicionários on-line na compreensão de textos em língua estrangeira. Resumo no VI Congresso Brasileiro de Lingüística Aplicada. Belo Horizonte: UFMG, 7-11 de outubro de 2001, p. 39

LELOUP Jean W.; PONTERIO, Robert. On the net. Vocabulary Support for Independent Online Reading. Language Learning \& Technology, v. 9, n.2, p. 3-7, May 2005. Disponible en: <http://lt.msu.edu/ vol9num2/net/> Acceso en: 1 dic. 2005.

LEU, Donald J. The new literacies: Research on reading instruction with the Internet and other digital technologies. In A.E. Farstrup \& S.J. Samuels (Eds.). What research has to say about reading instruction (3 ed.). Newark, DE: International Reading Association, 2002, p. 310-336.

Literacy and technology: Deictic consequences for literacy education in an information age. In M.L. KAMIL; P.B., et al. (Eds.). Handbook of reading research (v.3). Mahwah: NJ: Erlbaum, 2000, p. 743770.

LEU Jr., Donald J.; et al. Toward a Theory of New Literacies Emerging From the Internet and Other Information and Communication Technologies. In R.B. RUDDELL, \& N.J. UNRAU (Eds.). Theoretical Models and Processes of Reading Newark, DE: International Reading Association, 2004, p. 1570-1613

LEU, Donald J.; KINZER, Charles K. The convergence of literacy instruction with networked technologies for information and communication. Reading Research Quarterly, 35, p. 108-127, 2000.

LONG, Michael $H$. The role of linguistic environment in second language acquisition. In W. C. Ritchie \& T. K. Bhatia, (Eds.). Handbook of second language acquisition San Diego, CA: Academic Press, 1996, p. 413-468.

Focus on form: A design feature in language teaching methodology. In K. DeBot, R. Ginsberg, and C. Kramsch (Eds.). Foreign language research in crosscultural perspective. Amsterdam: John Benjamins, 1991, p. 39-52.

.; ROBINSON, P. Focus on form: Theory, research and practice. In DOUGHTY C.; WILLIAMS, J.

(Eds.). Focus on form in classroom second language acquisition. Cambridge: Cambridge University Press, 1998, p. 15-41. 
MARCUSCHI Luiz Antônio. O hipertexto como um novo espaço de escrita em sala de aula. Linguagem \& Ensino, v. 4, n. 1, p. 79-111, 2001. Disponible en:

<http://rle.ucpel.tche.br/php/edicoes/v4n1/f_marcuschi.pdf> Acceso en: 21 mayo 2005.

Linearização, cognição e referência: o desafio do hipertexto. In: Tercer Coloquio

Latinoamericano de Estudios del Discurso. Santiago de Chile, 5 a 9 de abril de 1999. Disponible en:: <http://www.filosofia.uchile.cl/Editorial/discurso_cambio/17Marcus.pdf> Acceso en: 11 dic. 2005.

NICOLSON, Roderick I.; FAWCETT, Angela J.; NICOLSON, Margaret K. Evaluation of a computer-based reading intervention in infant and junior schools. Journal of Research in Reading, v. 23, i. 2, p. 194-209, 2000.

PICA, Teresa. Research on negotiation: What does it reveal about second-language learning conditions, processes, and outcomes? Language Learning, v. 4, n. 3, p. 493-527, 1994.

RAMAL, Andrea Cecilia. Educação na cibercultura: hipertextualidade, leitura, escrita

e aprendizagem. Porto Alegre: Artmed, 2002.

SALABERRY, Rafael. The use of Technology for Second Language Learning and Teaching: A Retrospective. The Modern Language Journal, 85, pp. 39-56, 2001.

A theoretical foundation for the development of pedagogical tasks in Computer Mediated Communication. CALICO Journal, v. 14, n. 1, p. 5-34, 1996. Disponible en: <http://www.ruf.rice.edu/ rafaels/Salaberry1996CALICO.pdf> Acceso en 10 dic. 2005.

SAMPAIO, Isabel S.; SANTOS, Acácia A. Angeli dos. Leitura e redação entre universitários. Avaliação de um programa de intervenção. Psicologia em Estudo, Maringá, v. 7, n. 1, p. 31-38, jan.jun. 2002 Disponible en: <http://www.scielo.br/pdf/pe/v7n1/ v7n1a04.pdf> Acceso en 20 nov. 2005.

SCHMIDT, R. Deconstructing consciousness in search of useful definitions for applied linguistics. AILA Review, 11, 11-26, 1994.

The role of consciousness in second language learning. Applied Linguistics, v. 11, n. 2, p. 129-158, 1990.

SEDYCIAS, João. Por que os brasileiros devem aprender espanhol? In: (Org.). 0 ensino do espanhol em Brasil. São Paulo: Parábola Editorial, 2005, p. 35-44.

SELFE, Cynthia. Redefining Literacy: The Multilayered Grammars of Computers. In: HAWISHER, GAIL E. \& SELFE, C. (Eds.) Critical Perspectives on Computers and Composition Instruction. New York: Teachers College Press, p. 3-15, 1989.

SILVEIRA, Maria Inez Matoso. Modelos teóricos \& Estratégias de leitura. Suas implicações no ensino. Maceió: EdUFAL, 2005.

SWANSON, H.L.; DESHLER, D. Instructing Adolescents with Learning Disabilities: Converting a MetaAnalysis to Practice. Journal of Learning Disabilities. v. 36, n. 2, March/April, p. 124-135, 2003.

TANNER JACKSON, G.; GRAESSER, Arthur C. Aplicaciones del diálogo humano de tutoría al AutoTutor: Un sistema inteligente de tutoría. . Rev. Signos, v.39, n.60, p.31-48, 2006. Disponible en: <http:// www.scielo.cl/scielo.php?script=sci_arttext\&pid= S0718-09342006000100002\&lng=es\&nrm=iso> Acceso en: 9 marzo. 2006.

TOPPING, Keith J.; FISHER, Anna M. Computerised formative assessment of reading comprehension: field trials in the UK. Journal of Research in Reading, v. 26, i. 3, p. 267-279, 2003.

XAVIER, Antonio Carlos. Leitura, texto e hipertexto. In: Marcushi, L.A.; Xavier, A.C. (Orgs.) Hipertexto e gêneros digitais. Rio de Janeiro: Editora Lucerna, 2004, p. 170-180 


\section{ZARZOSA ESCOBEDO, Luis G. Programa de cómputo para el desarrollo de lectura estratégica a nivel universitario. Revista Digital UMBRAL 2000, 2004. Disponible en: <www.reduc.cl/reduc/}

zarzosa15.pdf> Acceso: 3 feb. 2006.

\section{Notas}

${ }^{1}$ Es de consenso que no existe un sólo letramiento y sí varios tipos y niveles de letramiento o multiletramientos (ver CASSANY, 2006).

2 En García Parejo (2005) podremos profundizar sobre el concepto de alfabetismo y la competencia de lectoescritura en general. En nuestro trabajo optamos por el término de letramiento en vez de alfabetización.

${ }^{3}$ Para ver definiciones, características e influencias del hipertexto, sugiero lecturas como Marcuschi (1999 y 2001), Ramal (2002) y Xavier (2004).

${ }^{4}$ Gran parte de su vasta producción puede ser consultada en la sección de publicaciones presente en el sitio web del proyecto http://www.autotutor.org/publications/pubs.htm

${ }^{5}$ A nuestro entender, éste es un buen ejemplo del principio de la "pedagogía de la pregunta" que Cárdenas (2004), inspirado en uno de los principios pedagógicos de Paulo Freire, propugna como de utilidad para la enseñanza de la lectura.

${ }^{6}$ Algo que es de gran interés en ese artículo de Zarzosa Escobedo (op. cit.), pero que no fue aquí presentado por escapar de los objetivos de este trabajo, es la clasificación de los diversos tipos de vicios que fueron observados en los estudiantes universitarios estudiados y que conducen a errores de comprensión de los textos.

${ }^{7}$ En las teorías interaccionistas de aprendizaje de segundas lenguas (GASS, 1997; LONG, 1996; PICA, 1994), los datos del input o output lingüístico tienen que ser captados o percibidos de forma consciente ("noticing") (SCHMIDT, 1990) para que ellos puedan ser convertidos en "intake" (o sea, sean incorporados a la memoria de trabajo), lo cual no necesariamente garantiza que quede en la interlengua del alumno. Schmidt (1994 apud ELLIS, 2006), hipotetiza que esa "captación" o "noticing" es una condición necesaria para la adquisición. Por otro lado, es mucho más probable que sea percibida la forma lingüística durante la interacción y una respuesta activa del sujeto puede indicar que fue percibido ese input. El "uptake" del alumno, definido como aquellas manifestaciones observables provocadas por las respuestas dadas por el profesor (o computadora en este caso), y el "foco en la forma", como la atención durante la interacción debido a algún problema percibido durante la comprensión o producción (LONG, 1991; LONG y ROBINSON, 1998, p. 23) son otros de los conceptos teóricos de utilidad en nuestro caso.

${ }^{8}$ Ver en los artículos de Leffa (2003a y 2003b) más detalles sobre los tipos de retroalimentación y su funcionamiento en el sistema de autor "ELO".

${ }^{9}$ En este ejemplo, las preguntas, respuestas y explicaciones aparecen en la pantalla del programa siempre en español.

${ }^{10}$ La palabra portuguesa "dica", significa sugerencia o recomendación en español.

${ }^{11}$ Como la actividad con ese texto quedó disponible en la Internet, fue pedida autorización al autor para su uso y divulgación, la cual fue concedida.

${ }^{12}$ La actividad puede ser vista en http://gonzalo.cedu.ufal.br/curso/porque_aprender_espanhol.htm

13 Es necesario aclarar que la mayoría de las lecturas realizadas en ese curso fueron de textos en lengua española o traducidos a esa lengua, pues partimos del presupuesto de que la realización de las lecturas académicas en español contribuirían con un mayor contacto y, presumiblemente, con una mayor adquisición de esa lengua por parte de los futuros profesores. En este caso, la lectura analizada fue realizada en portugués por varias razones: era el primer texto estudiado en el curso de prácticas de enseñanza y también no queríamos obstaculizar en la emergencia de nuevas ideas sobre el por qué es importante el estudio de la lengua española, como fue discutido en otras clases. De cualquier forma, hubo un input en español pues las preguntas, opciones, aclaraciones y respuestas alrededor del texto estaban escritas en español, cosa que estimamos que contribuyó de forma positiva en su adquisición, como veremos más adelante, en este trabajo.

${ }^{14}$ Realizado por medio de un formulario CGI para envío automático por Internet al autor.

${ }^{15}$ Esas frases fueron emitidas en portugués: "Ainda bem. Depois de quatro anos estudando aqui, por primeira vez viemos no laboratório!", "Que legal isto!", "No acredito. Vamos usar os computadores?"

${ }^{16}$ Para una mayor divulgación y aplicabilidad educacional, estas actividades de lecturas, desarrolladas con el sistema "ELO", pueden ser fácilmente preparadas en forma de Objetos de Aprendizaje Reutilizables (OAR).

17 Los procedimientos recomendados por Silveira (2005, p. 136-7) pueden ayudar en las acciones futuras para el desarrollo de prácticas de lectura eficaces.

${ }^{18}$ El efecto Hawthorne, debe su nombre a la fábrica de la Western Electric Company, donde los investigadores encontraron y describieron ese efecto, en las décadas de los años veinte y treinta del siglo pasado. Ese efecto no es más que la respuesta positiva y posible elevación de la autoestima ante la novedad y las acciones consideradas como positivas realizadas por otros. 


\section{INFORMÁTICA NA EDUCAÇÃO:}

teoria \& prática Porto Alegre, v.9, n.1, jan./jun. 2006. ISSN 1516-084X

Recebido em 11 de julho de 2005

Aceito para publicação em 17 de outubro de 2005

\section{Agradecimientos}

Quiero agradecer los comentarios a la versión inicial de este artículo, realizados por Vilson Leffa y tres revisores anónimos.

\section{Gonzalo Enrique Abio Vírsida}

Máster em Estudos da Linguagem

Centro de Educação, Universidade Federal de Alagoas

E-mail: gonzalo@cedu.ufal.br 
